\title{
Enhancement of Contour Using Laser Scar Treatment in the One- Stage Nasal Ala Reconstruction with Nasolabial Fold Flap
}

\author{
Sun Jae Lee \\ Eun Soo Park
}

Department of Plastic and Reconstructive Surgery, College of Medicine, Soonchunhyang University, Bucheon, Korea
Received June 18, 2018

Accepted June 22, 2018

\section{Correspondence}

Eun Soo Park

Department of Plastic and Reconstructive Surgery, Soonchunhyang University, Colleage of Medicine, Bucheon Hospital, 170 Jomaru-ro,

Wonmi-gu, Bucheon 14584, Korea

Tel.: +82-32-621-5319

Fax: +82-32-621-5662

E-mail: peunsoodschcm.ac.kr

(C) Korean Society for Laser Medicine and Surgery

(c) This is an open access article distributed under the terms of the Creative Commons Attribution NonCommercial License (http://creativecommons.org/ licenses/by-nc/4.0) which permits unrestricted noncommercial use, distribution, and reproduction in any medium, provided the original work is properly cited.
Postoperative scarring around the nasolabial area and alar rim poses very high cosmetic concerns, leading to patient dissatisfaction. Several strategies, like laser therapy, have been developed to improve its clinical aspects. However, to date, little is known about when in the early wound healing phases laser exposure would have the most optimal results. This paper introduces a remarkable case of effectively treating a 32-year-old male patient with scarring on the alar rim and nasolabial fold using early active laser treatment. We started carbon dioxide $\left(\mathrm{CO}_{2}\right)$ laser therapy early in the second month after surgery. In the course of laser treatment, we performed a combined treatment using an ablative fractional $\mathrm{CO}_{2}$ laser device and the combination of pulsed-dye laser and neodymium: yttrium-aluminum-garnet laser. A total of 7 sessions of $\mathrm{CO}_{2}$ laser therapy and 3 sessions of cynergy laser therapy were performed, and the patient was followed-up for evaluation. These early laser treatments resulted in both patient and clinical satisfaction. In this case, we confirmed that early treatment with $\mathrm{CO}_{2}$ laser and cynergy laser can have a positive effect on scarring of the alar rim and nasolabial fold.

\section{Key words}

Nasal ala contour; Fractional carbon dioxide laser; Cynergy; Multiflex; Early laser therapy 


\section{INTRODUCTION}

The scars around the nasolabial area and the alar rim have very important cosmetic concerns. There are various studies reporting about the reconstruction of the alar defects caused by trauma or tumor. The reconstruction of the full-thickness defects involving the alar cartilage and mucosa may present difficulties because of the complex 3-dimensional structure of the alar region. ${ }^{1}$ After reconstructions performed using inappropriate methods, functional problems including the collapse of the external nasal valve and esthetic problems such as retraction in the alar wing or asymmetry may develop. Therefore, reestablishing the 3-dimensional structure of the nasal ala is of utmost importance in the reconstruction to be performed. One of the effective classic methods used in nasal alar reconstructions is the nasolabial flap. Thanks to the laxity of the cheek tissue, full-thickness alar reconstructions can be performed using nasolabial flaps. The flap donor area is primarily repaired, and the scar is aligned with the nasolabial fold. ${ }^{2}$

Especially after the surgery of the nose, the scar can make the patient suffer from cosmetic difficulties and reduce social confidence. Multiple strategies have been developed to prevent or improve postoperative surgical scarring, including silicone sheets, topical corticoids, dermabrasion, and laser therapy. ${ }^{3}$ Over the past decade, laser skin resurfacing with carbon dioxide $\left(\mathrm{CO}_{2}\right)$ and erbium (Er):yttrium-aluminumgarnet (YAG) lasers has become popular for the treatment of scars. The ablative fractional $\mathrm{CO}_{2}$ laser combines the concept of fractional photothermolysis with an ablative 10,600 nm wavelength. ${ }^{4,5}$ However, little is known on when in the early wound healing phases laser exposure most optimally should be provided.

\section{CASE REPORT}

This case is a 30-year-old male patient with a skin defect of the alar rim caused by direct trauma. The patient underwent a subcutaneous pedicle nasolabial island flap to reconstruct the nasal defects. The flap to be elevated from the nasolabial fold was drawn with its width equaling the height of the defect and its height at least twice as long as the width of the defect. The flap donor area (nasolabial fold) closed with primary suture (Fig. 1, 2).

Two months after the flap operation, the patient started an early laser treatment for scar treatment. We used two laser machines, an ablative fractional $\mathrm{CO}_{2}$ laser device (LineXel ${ }^{\circledR}$; UTI Co. Ltd, Seoul, Koreal and the combina- tion of pulsed-dye laser (PDL) and neodymium (Nd):YAG laser (Cynergy with MultiPlex; Cynosure, Westford, MA, USA) which allows the sequential emission of each wavelengths. Treatment was accomplished with a 10,600-nm fractional $\mathrm{CO}_{2}$ laser using laser parameters were set as a pulse duration of 280-400 miliseconds and a distance of 1.0 milimeters. During $\mathrm{CO}_{2}$ laser treatment, cynergy treatment was performed simultaneously. Cynergy was combination treated at 2, 4, and 6th session in $\mathrm{CO}_{2}$ laser treatment. We used multiflex mode among various modes of Cynergy. The $595 \mathrm{~nm}$ pulsed-dye laser was fired first with a pulse duration of $10 \mathrm{~ms}$ and fluence of $7 \mathrm{~J} / \mathrm{cm}^{2}$. The long-pulsed 1,064 nm Nd:YAG laser was fired later at $15 \mathrm{~ms}$ pulse, $40 \mathrm{~J} / \mathrm{cm}^{2}$ using a $7-\mathrm{mm}$ spot size with a medium delay between the two pulses. The therapeutic endpoint was decided to immediate whitening following laser irradiation. Energy densities were reduced if tissue bleeding was prominent. Patients were treated up to 7 sessions, at intervals ranging from 4 weeks. A single physician performed all treatments. Topical lidocaine cream (EMLA; AstraZeneca Canada Inc., Ontario, Canada) was applied 60 minutes before treatment. After treatment, the patients were asked to apply topical fusidic acid $2 \%$ under occlusion until the supeficial wound was closed and to avoid sun exposure. We photographed the patient's progress with each laser treatment. Clinical photos were obtained under standardized lighting conditions and body positioning using a digital camera (Fig. 3-5).

The patient showed a remarkable improvement of the surgical scar through laser treatment. Especially, it is meaningful that a good effect was obtained through the combination therapy of two lasers. After laser treatment, the patient had a good effect on the appearance of the alar rim as well as the treatment of the scar. The current case demonstrates clear clinical efficacy in the treatment of facial scars with fractional $\mathrm{CO}_{2}$ laser. Based on these results, we recommend early intervention in facial scars, to maximize the clinical benefit and to mitigate social stigmata as early as possible.

\section{DISCUSSION}

Laser treatments are established procedures to improve clinical appearance of mature scars. ${ }^{6}$ Within recent years, a preventative approach of minimizing scar formation by applying laser during the wound healing process has increasingly been adopted. Studies on early laser intervention indicate promising response in a range of scarcharacteristics, including improved pliability, smoother surface and reduced scar thickness. ${ }^{7}$ A variety of termina- 

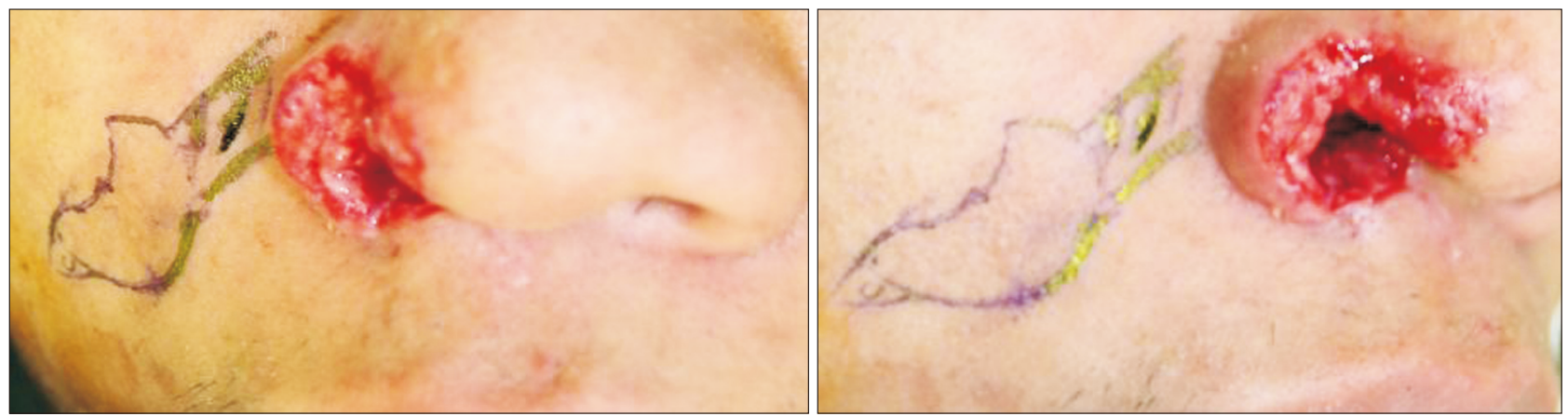

Fig. 1. Pre-operative photographic finding and flap design.
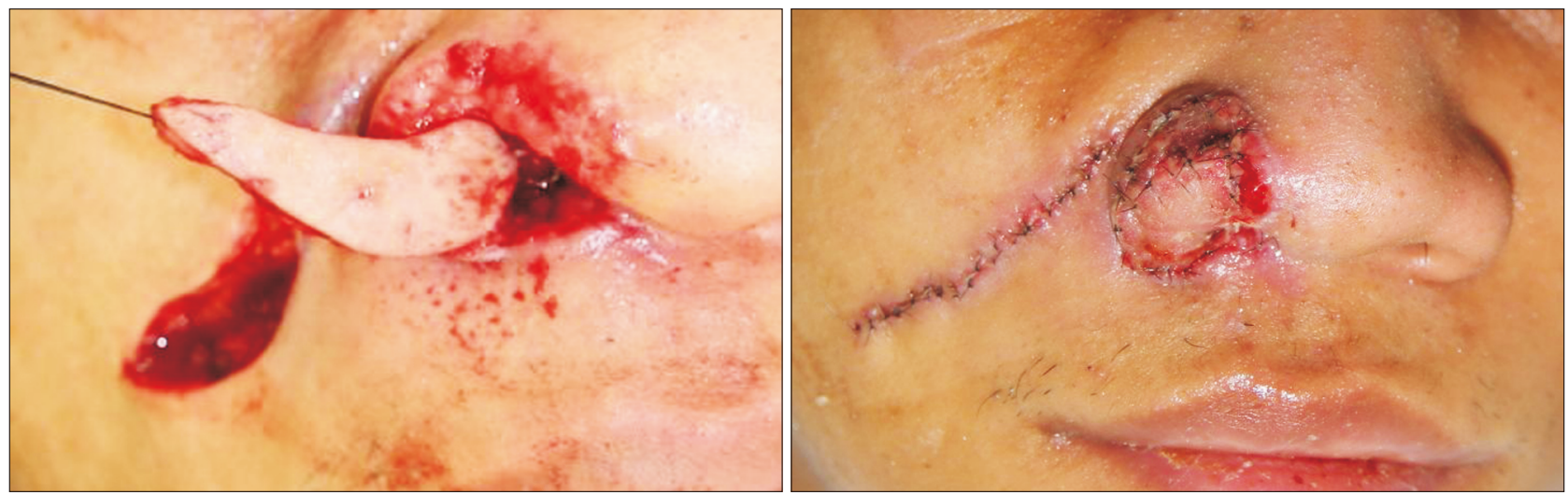

Fig. 2. Intra-operative flap elevation and immediately post-operative photographic finding.
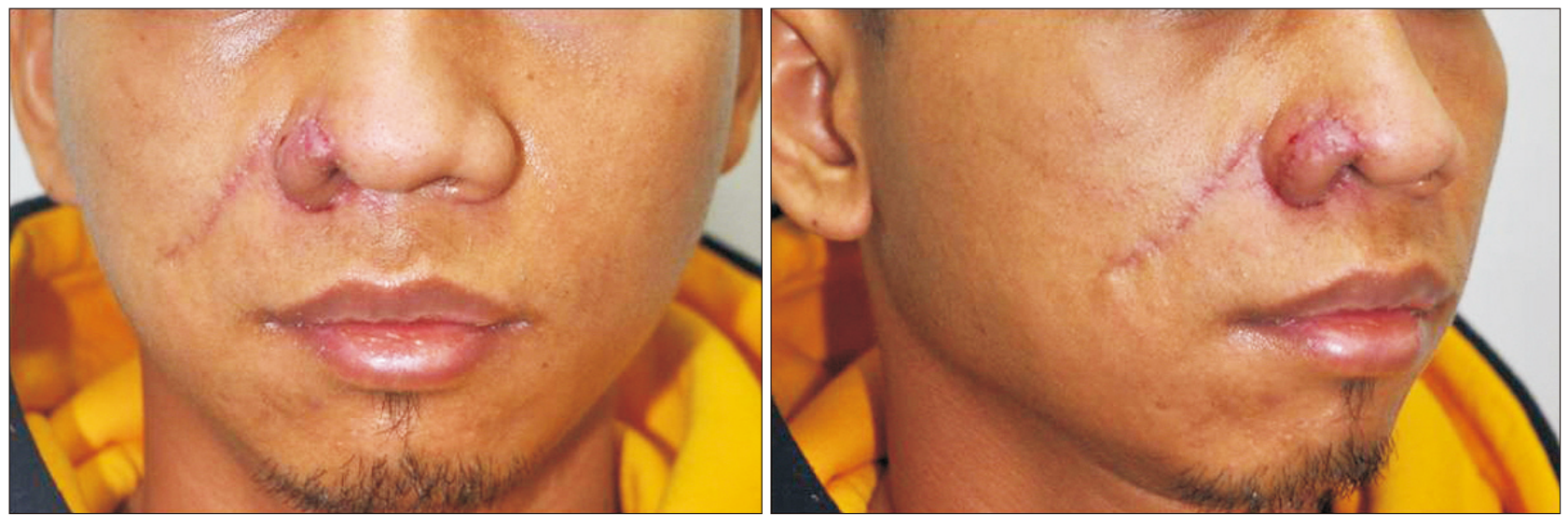

Fig. 3. One-month postoperative photographs of 30-year-old male who underwent nasolabial flap operation.

tion lasers can be used for scar treatment, ablative fractional lasers are an effective treatment modality for facial scars after reconstruction of skin cancer defects. ${ }^{8}$

Natural wound healing is divided into three overlapping phases, beginning with the inflammation phase (0-4 days), shifting towards the proliferation phase (4-21 days) and progressing to the remodeling or maturation phase (>21 days) lasting up to 24 months. Laser exposure specifically targeting the early phases of wound healing may be the key to scar minimization. Furthermore, as the acute inflammatory response induced by laser peaks after 24 hours, fractional laser-exposure 1 day prior to wounding could ensure the presence of stress protective cytokines in the skin at the time of wounding. As a result, 

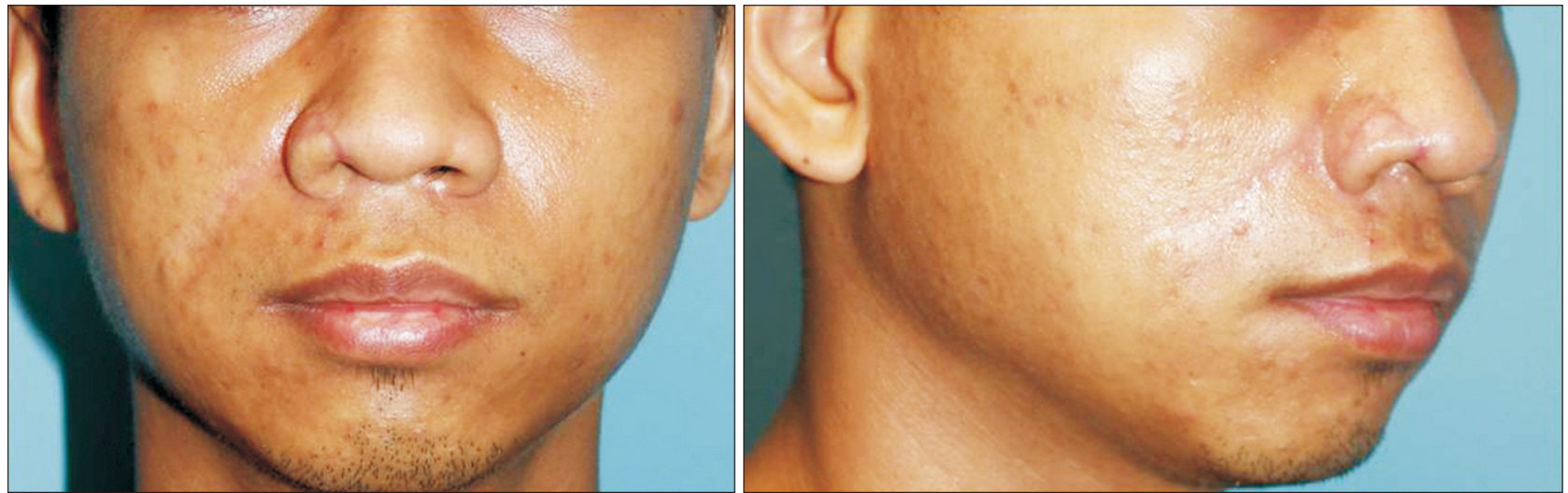

Fig. 4. Six months after started laser treatment photographic finding (postop, 8 months).
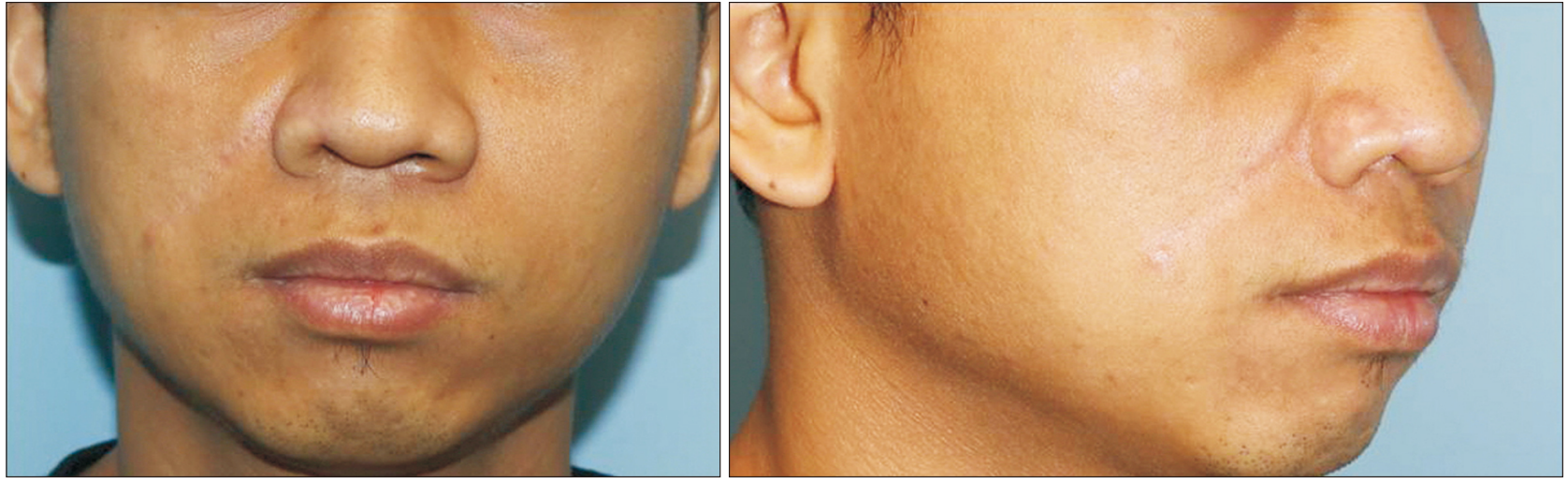

Fig. 5. After all laser treatments have been done follow-up photographs (postop, 1 year).

more efficient scar prevention could be expected. Laserpriming of the skin prior to elective surgical procedures may thus limit scar formation.

The body of evidence on early laser intervention comprises less than 30 studies applying laser within 3 months after injury. Thirteen of these studies were randomized controlled trials. ${ }^{9,10}$ Sixteen studies applied laser in the proliferation phase of wound healing (4-21 days postinjuryl, generally performed at the point of suture removal using PDL, fractional laser. Overall, these studies report improved subsequent scar appearance. ${ }^{11,12}$ Only Hong et al..$^{13}$ investigated the appropriate timing of laser intervention to reduce scarring. This study shows that the use of early fractional $\mathrm{CO}_{2}$ lasers 4 weeks after surgery or trauma is an effective and safe method to minimize scar formation, ${ }^{13}$ substantiating the concept of "the earlier the treatment, the better."

Similar to this study, clinical investigations of early laser treatment to reduce scar formation have previously used single laser exposure of various types. ${ }^{12}$ Under these conditions, slight laser-mediated improvement of treated scars has been reported. In accordance with previous results, subtle differences were visualized in this study. ${ }^{14}$ Using multiple laser treatments, more prominent clinical results have been reported in preceding studies. Thus, applying laser over several treatment sessions may offer a more pronounced reduction of scar formation.

Various reconstruction methods including local flaps, forehead flaps, composite grafts, and free flaps have been reported to be used in the repair of alar defects. ${ }^{15}$ The defect size, symmetry, color harmony with the surrounding tissues, skin texture, and donor area are important factors to be considered in nasal alar reconstructions. Because of its closeness to the nasal ala, the nasolabial region is among the most suitable donor areas in terms of color and texture. One of the oldest classic methods used in nasal alar reconstructions is the nasolabial flap. Since its first use in nasal reconstructions, various modifications of this flap have been reported. The scar in the flap donor area is much less visible compared with various methods including forehead flaps. Thanks to cheek skin mobility, full-thickness nasal alar defects can be closed 
in 1 session in patients; no revision is needed around the flap pedicle, and donor area morbidity is reduced. Thus, the flap can be planned in the desired form and size, and a total alar reconstruction can be realized.

Different techniques are used to enhance wound healing, but, thanks to the rapid advances in laser technology, a new method has emerged with a promising future for modern wound healing lasers have also been applied to improve the appearance of such scars. The first lasers utilized for this purpose were ablative, nonselective lasers: $\mathrm{CO}_{2}$ or Er:YAG laser ${ }^{4,5}$ The flashlamp-pumped PDL, which emits light at a wavelength of $585 \mathrm{~nm}$, has become the gold standard in the treatment of port-wine stains and also an effective treatment modality for superficial vascular lesions, including those associated with photoaging, such as facial telangiectasias. The Nd:YAG laser emits light at a wavelength of 1,064 nm, which allows deep penetration into the dermis and vascular specificity due to a broad absorption peak of oxyhemoglobin above 800 $\mathrm{nm} .{ }^{16,17} \mathrm{PDL}$ and Nd:YAG lasers of different wavelengths have been incorporated in the novel dual laser device built into the same console. Laser and light source combinations are currently being examined for their complementary, additive, or sometimes synergistic action. The combined laser therapy additionally decreases the accumulation of fibroblast cells in the scar tissue and stimulates the production of reticular collagen fibers. Krisztina Vas et al, one half of each of the postoperative linear scars immediately after suture removal was treated three times at monthly intervals with the combined 585/1,064 nm laser and the cosmetic appearances of the treated and untreated scar halves were compared. ${ }^{18}$

In this patient case, we combined the effects of the known $\mathrm{CO}_{2}$ laser with the effect of the Cynergy. It was also confirmed that early treatment with laser treatment could have a good effect on scar treatment. Therefore, selecting an appropriate laser and performing active scar treatment early may have a positive impact on the prognosis of the patient. It is common to reconstruct the entire subunit during reconstruction in plastic surgery. In this case, it is significant that the contour enhancement was achieved through the use of remaining natural alar grooves and the subsequent laser treatment.

\section{REFERENCES}

1. Castello JR, Taglialatela Scafati S, Sánchez O. Bilateral nasal ala reconstruction of the cocaine-injured nose with 2 free reverse-flow helical rim flaps. Ann Plast Surg 2014;73:304-6.

2. Durgun M, Özakpınar HR, Selçuk CT, Sari E, Seven E, Inözü
E. Repair of full-thickness nasal alar defects using nasolabial perforator flap. Ann Plast Surg 2015;75:414-7.

3. Monstrey S, Middelkoop E, Vranckx JJ, Bassetto F, Ziegler UE, Meaume $S$, et al. Updated scar management practical guidelines: non-invasive and invasive measures. J Plast Reconstr Aesthet Surg 2014;67:1017-25.

4. Elsaie ML, Choudhary S. Lasers for scars: a review and evidence-based appraisal. J Drugs Dermatol 2010;9:1355-62.

5. Berlin AL, Hussain M, Phelps R, Goldberg DJ. A prospective study of fractional scanned nonsequential carbon dioxide laser resurfacing: a clinical and histopathologic evaluation. Dermatol Surg 2009;35:222-8.

6. Vrijman C, van Drooge AM, Limpens J, Bos JD, van der Veen JP, Spuls PI, et al. Laser and intense pulsed light therapy for the treatment of hypertrophic scars: a systematic review. Br J Dermatol 2011;165:934-42.

7. Nouri K, Jimenez GP, Harrison-Balestra C, Elgart GW. 585 -nmpulsed dye laser in the treatment of surgical scars starting on the suture removal day. Dermatol Surg 2003;29:6573.

8. Cho SE, Nam SM, Kim YB, Park ES. Local flap surgical scar management caused by skin cancer using fractional $\mathrm{CO} 2$ laser treatment. Arch Aesthetic Plast Surg 2015;21:18-22.

9. Rohrer TE, Ugent SJ. Evaluating the efficacy of using a shortpulsed erbium:YAG laser for intraoperative resurfacing of surgical wounds. Lasers Surg Med 2002;30:101-5.

10. Tierney E, Mahmoud BH, Srivastava D, Ozog D, Kouba DJ. Treatment of surgical scars with nonablative fractional laser versus pulsed dye laser: a randomized controlled trial. Dermatol Surg 2009;35:1172-80.

11. Conologue TD, Norwood C. Treatment of surgical scars with the cryogen-cooled $595 \mathrm{~nm}$ pulsed dye laser starting on the day of suture removal. Dermatol Surg 2006;32:13-20.

12. Alam M, Pon K, Van Laborde S, Kaminer MS, Arndt KA, Dover JS. Clinical effect of a single pulsed dye laser treatment of fresh surgical scars: randomized controlled trial. Dermatol Surg 2006;32:21-5.

13. Hong SC, Park ES, Kim YB, Nam SM. Effects of minimizing scar formation by early fractional $\mathrm{CO} 2$ laser resurfacing. Arch Aesthetic Plast Surg 2014;20:109-13.

14. Lee SH, Zheng Z, Roh MR. Early postoperative treatment of surgical scars using a fractional carbon dioxide laser: a splitscar, evaluator-blinded study. Dermatol Surg 2013;39:1190-6.

15. Selçuk CT, Ozalp B, Durgun M, Bozkurt M, Baykan H. Reconstruction of full-thickness nasal alar defects using cartilagesupported nonfolded nasolabial flaps. J Craniofac Surg 2012;23:1624-6.

16. Berlin AL, Hussain M, Goldberg DJ. Cutaneous photoaging treated with a combined 595/1064 nm laser. J Cosmet Laser Ther 2007;9:214-7. 


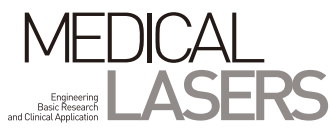

17. Trelles MA, Weiss R, Moreno-Moragas J, Romero C, Vélez M, Alvarez $X$. Treatment of leg veins with combined pulsed dye and Nd:YAG lasers: 60 patients assessed at 6 months. Lasers Surg Med 2010;42:609-14.
18. Vas K, Gaál M, Varga E, Kovács R, Bende B, Kocsis A, et al. Effects of the combined PDL/Nd:YAG laser on surgical scars: vascularity and collagen changes evaluated by in vivo confocal microscopy. Biomed Res Int 2014;2014:204532. 